\title{
Dispersão de sementes de Copaifera arenicola (Caesalpinioideae-Fabaceae) por formigas cortadeiras em remanescentes de Caatinga
}

\author{
Dráuzio Correia Gama ${ }^{1}$, Favízia Freitas de Oliveira ${ }^{2}$, Caroline Tito Garcia ${ }^{2}$, José Monteiro do Nascimento Júnior ${ }^{3}$ \\ ${ }^{1}$ Universidade Estadual do Sudoeste da Bahia-UESB, Estrada Bem Querer, Km-04 - 3293, 3391, Campus de Vitória da Conquista - BA, 45083-900 \\ ${ }^{2}$ Universidade Federal da Bahia-UFBA, Rua Barão de Jeremoabo, 668, Campus Universitário de Ondina, Salvador - BA, 40170-115 \\ ${ }^{3}$ Universidade Estadual da Bahia-UNEB, Campus VIII; Rua da Gangorra, 503, General Dutra, Paulo Afonso - BA, 48608-240
}

*Autor para correspondência: drauziogama@ hotmail.com

Recebido: Março 2018 / Aceito: Dezembro 2019 / Publicado: Dezembro 2019

\section{Resumo}

O presente estudo registra o processo mirmecórico de sementes da espécie arbórea Copaifera arenicola (Ducke) J. Costa \& L. P. Queiroz por formigas-cortadeiras da espécie Atta opaciceps Borgmeier, 1939 (Hymenoptera: Formicidae), observado em área de remanescente de Caatinga, localizada no município de Ribeira do Pombal, no nordeste do Estado da Bahia. Foi observado um formigueiro de Atta opaciceps contendo sementes de Copaifera arenicola em seu interior, o qual distava quatro metros da planta-mãe, tendo sido observadas na trilha de dispersão formada pelas formigas algumas sementes abandonadas e outras sementes da mesma planta sendo transportadas por espécimes de Atta opaciceps. Durante o período de estudo, nenhuma outra espécie de formiga foi observada em contato ou carregando as sementes de Copaifera arenicola. O que merece destaque aqui é o comportamento das formigas, por cortarem o tegumento da semente antes de transportá-la, além do tamanho avantajado da semente em relação ao tamanho do inseto. Foi observada também uma plântula de Copaifera arenicola encontrada a dois metros do formigueiro, o que pode sugerir a ação das formigas na dispersão espacial dessa espécie botânica.

Palavras-chave: Espécie arbórea; mirmecoria; Atto opaciceps; semiárido.

\begin{abstract}
The present study registers the myrmecochory seeds dispersal process of the arboreal species Copaifera arenicola (Ducke) J. Costa \& L. P. Queiroz by leafcutter ants Atta opaciceps Borgmeier, 1939 observed in a Caatinga remnant area, located in the municipality of Ribeira do Pombal, northeast of the Bahia State, Brazil. It was observed a nest of Atta opaciceps containing Copaifera arenicola seeds in its interior, which was located four meters away from the mother plant, and observed at the ants dispersal trail some abandoned seeds and other seeds of the same plant being transported by Atta opaciceps specimens. During the study period, no other ant species were observed in contact with or carrying Copaifera arenicola seeds. What is noteworthy here is the ant's behavior of cutting the seed integument before transporting it, in addition to the larger size of the seed related to the size of the insect. It was also observed a Copaifera arenicola seedling found two meters from the anthill, which may suggest the ant's action in the spatial dispersal of this botanical species.
\end{abstract}

Keywords: Tree species; myrmecochory; Atta opaciceps; semiarid.

\section{Introdução}

O processo de dispersão de diásporos por animais envolve relações específicas entre esses elementos vegetais e agentes dispersores (Deminicis et al. 2009). Dentre os vetores zoocóricos de sementes, têm-se as formigas que atuam nos ecossistemas com ampla distribuição geográfica, abundância local elevada e alta riqueza de espécies, assumindo um papel de grande relevância como dispersores secundários, incluindo a maior parte das espécies de formigas forrageando frutos e sementes na superfície do solo (Queiroz et al. 2006; Farnese et al. 2011; Oliveira et al. 2014).

As interações entre formigas e plantas não mirmecóricas (cujas sementes não estão adaptadas para a dispersão por formigas) são pouco documentadas (Farnese et al. 2011), tendo sido mais estudadas nas regiões áridas da África e Austrália e, muito pouco documentado na América do Sul (Costa et al. 2007), sendo que os poucos registros no Brasil foram realizados em alguns ecossistemas de Florestas, Cerrado, Restinga e Caatinga (Costa et al. 2007), com destaque às formigas-cortadeiras.

Em sementes, algumas características morfológicas assumem importância por potencializarem a dispersão em diferentes tipos de mecanismos zoocóricos e distâncias, independentemente da forma de ocorrência desse processo (Deminicis et al. 2009), como o descolamento de elaissomos e a escarificação de sementes por formigas cortadeiras, que são dois comportamentos de manipulação podendo ser fundamentais na germinação de sementes (Fernandes et al. 2018).

Dentre as formigas-cortadeiras, merecem destaque aquelas do gênero Atta Fabricius, 1804 (Hymenoptera: Formicidae), reconhecidas popularmente como saúvas. Esse gênero corresponde a 15 espécies pertencentes à tribo Attini Smith, 1858, onde habitam ninhos subterrâneos e se alimentam de fungos cultivados em partes de plantas, inclusive sementes (Hölldobler e Wilson 1990; Autuori 2010).

Autores como Corrêa et. al. (2010), Meyer et al. (2011), Hardesty (2011) e Oliveira et al. (2014), ressaltam que em florestas neotropicais a manipulação e a dispersão de sementes por formigas-cortadeiras, em especial do gênero Atta spp., pode modificar as taxas de germinação e o recrutamento de plântulas, atuando, direta ou indiretamente, como um filtro para o estabelecimento de espécies ou grupos ecológicos, alterando a estrutura florística, a distribuição espacial das plantas e a forma funcional das populações, principalmente quando em torno de seus ninhos influenciando diretamente a composição da vegetação.

Segundo Oliveira et al. (2014), as formigas podem servir como bioindicadores, com respostas rápidas ao stress do meio, no que se refere a alterações do ambiente, sendo importantes também por agirem contribuindo para boa qualidade do solo, a exemplo da Atta sexdens (Linnaeus 1758), cujos ninhos, conforme Moutinho, Nepstad e Daividson (2003), podem alcançar $3 \mathrm{~m}$ de profundidade, proporcionando efeitos positivos nas propriedades do solo, pela maior aeração em decorrência da porosidade e disponibilidade de nutrientes pelo revolvimento e ingresso de biomassa vegetal. 
Nesse contexto, o presente trabalho tem por objetivo registrar a dispersão de sementes de Copaifera arenicola (Ducke) J. Costa e L. P. Queiroz por formigas cortadeiras em remanescentes de caatinga no município de Ribeira do Pombal, Bahia.

\section{Material e Métodos}

A região de estudo compreende um fragmento arbóreoarbustivo de caatinga com área de 21 ha e altitude média de $227 \mathrm{~m}$ (coordenadas: Lat $10^{\circ} 51^{\prime} 45^{\prime \prime} \mathrm{S}$ e Long $38^{\circ} 33^{\prime} 29^{\prime \prime} \mathrm{W}$ ), localizado no município de Ribeira do Pombal, Bahia (Gama e Jesus 2018).

O solo é do tipo Latossolo Vermelho-Amarelo arenoso, profundo e pouco fértil. O clima é do tipo BSw'h', considerado semiárido quente e seco, segundo a classificação de Köppen, e com índice pluviométrico médio de 711 mm.ano ${ }^{-1}$ e temperatura média de $24,2{ }^{\circ} \mathrm{C} . \mathrm{ano}^{-1}$, fazendo parte da ecorregião do Raso da Catarina de Domínio Fitogeográfico da Caatinga (Gama e Jesus 2018).

A espécie arbórea Copaifera arenicola (Ducke) J. Costa e L. P. Queiroz pertencente ao gênero Copaifera L. (Caesalpinioideae-Fabaceae), podem atingir até $10 \mathrm{~m}$ de altura. É endêmica de solos profundos sedimentar com exclusiva ocorrência confirmada nos Estados da Bahia, Ceará, Pernambuco e Rio Grande do Norte, em Domínio Fitogeográfico da Caatinga (Gama e Nascimento Júnior 2019).

Botanicamente é reconhecida pelas suas folhas glabras. folíolos que variam entre 2-3 pares, forma laminar oval com ápice agudo e consistência coriácea, inflorescência em panícula de tamanho até uma vez maior que a folha adiacente. flores contendo sépalas glabras somente externamente, fruto do tipo legume com formato levemente falcado e contendo uma semente ovóide por fruto, esta apresentando arilo (Gama e Nascimento Júnior 2019).

Através de caminhadas em transecto no período de agosto de 2015 , entre às 9 h00 e 11 h00 da manhã, e com auxílio de máquina fotográfica digital, registrou-se o comportamento de coleta e transporte das sementes de Copaifera arenicola realizado pelas formigas, tendo sido alguns espécimes capturados e encaminhados ao Laboratório de Bionomia, Biogeografia e Sistemática de Insetos (BIOSIS) do Instituto de Biologia, unidade associada ao Museu de História Natural da Universidade Federal da Bahia-UFBA (MHNBA/MZUFBA), para identificação, com confirmação da espécie realizada por taxonomista especialista em Formicidae (Hymenoptera), tendo sido os espécimes depositados no acervo entomológico do MHNBA.

Para os estudos morfológicos, os espécimes de formigas coletados foram identificados e fotografados com uso de microscópio estereoscópico Leica M165C, acoplado com câmera digital Leica DFC295, montadas em fotomontagem através do software Leica Application Suite V4.1 Interactive Measurements, Montage.

\section{Resultados e discussão}

Foram observadas sete árvores adultas de Copaifera arenicola (Ducke) J. Costa \& L. P. Queiroz (Fabaceae), sendo duas em frutificação e cinco árvores sem frutos, mas apresentando sementes caídas. As árvores com sementes caídas encontravam-se a quatro metros de distância. aproximadamente, de um ninho de formigas-cortadeiras Atta opaciceps Borgmeier, 1939 (Figura 1).
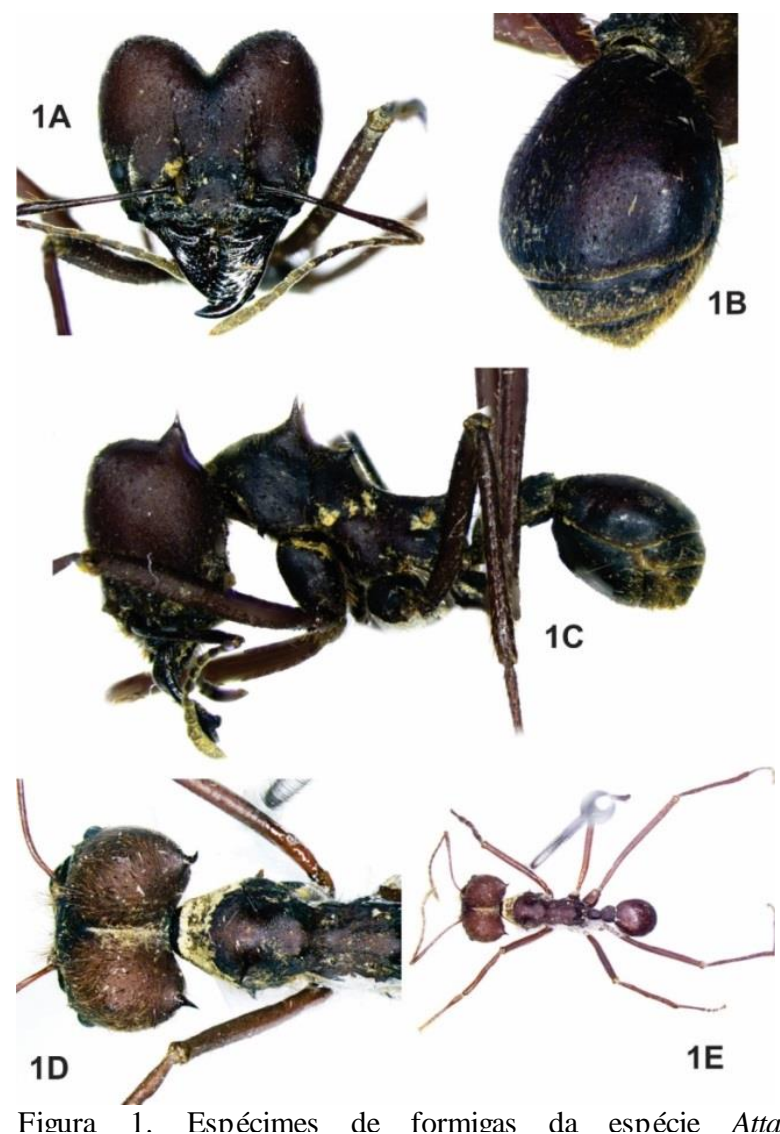

Figura 1. Espécimes de formigas da espécie Atta opaciceps Borgmeier, 1939 (Hymenoptera, Formicidae), coletados no município de Ribeira do Pombal (Bahia), quando transportando sementes de Copaifera arenicola (Ducke) J. Costa \& L. P. Queiroz (Fabaceae): 1A a 1COverária Maior: 1D e 1E - Overária Média. 1A- Cabeca em vista frontal; 1B- Abdome em vista latero-dorsal; 1C- Corpo em vista lateral; 1D- Cabeça em vista polar; 1E- Corpo em vista dorsal.

$\mathrm{Na}$ trilha de dispersão formada pelas formigas, em direção ao formigueiro, foram encontradas sementes de $C$. arenicola abandonadas e outras sementes sendo transportadas por A. opaciceps, além de algumas sementes apresentando parte do seu tegumento retirado pelas formigas (Figura 2). Não foi registrada nenhuma outra espécie de formiga presente no local que estivessem em contato ou transportando as sementes de $C$. arenicola, além da $A$. opaciceps. Alguns desses formigueiros continham sementes armazenadas em seu interior, trazidas pelas formigas com parte do tegumento retirado (Figura 3 ).

Foi encontrada uma plântula de Copaifera arenicola aproximadamente a dois metros de um dos formigueiros observados (Figura 3B), fato que também foi constatado por Teixeira et al. (2007) em ambiente de restinga, o que pode ter sido resultado do processo de dispersão proporcionado pelas formigas.

A dispersão de sementes de Copaifera arenicola por formigas é um caso raro, não havendo registro na literatura de nenhuma dispersão desse tipo para a referida espécie. Sendo ainda um fato desconhecido no Bioma Caatinga a dispersão de sementes realizada por formigas cortadeiras, com nenhum registro ainda dessa natureza, com registro prévio apenas do comportamento forrageiros dos gêneros Atta sp. e Acromyrmex sp. como importantes herbívoros no bioma (Leal et al. 2007; Leal et al. 2018). 
Gama et al.

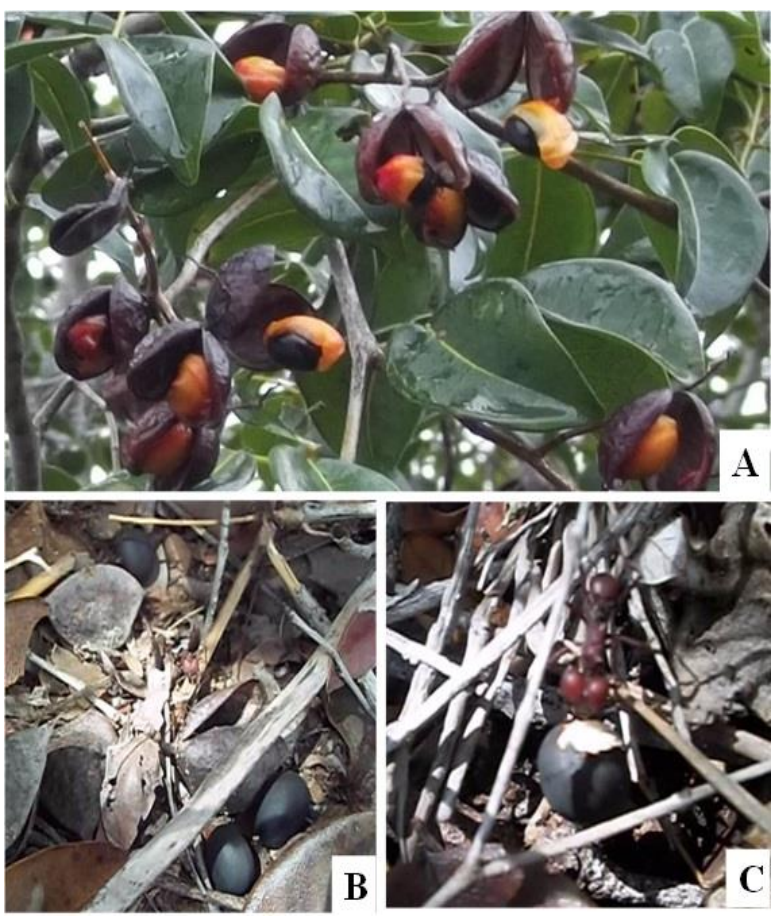

Figura 2. Copaifera arenicola (Ducke) J. Costa \& L. P. Queiroz (Fabaceae) em remanescente de caatinga, município de Ribeira do Pombal-BA: A- Frutos com semente e arilo: B- Sementes caídas sem arilos; C- Atta opaciceps Borgmeier, 1939 retirando tegumento de semente.
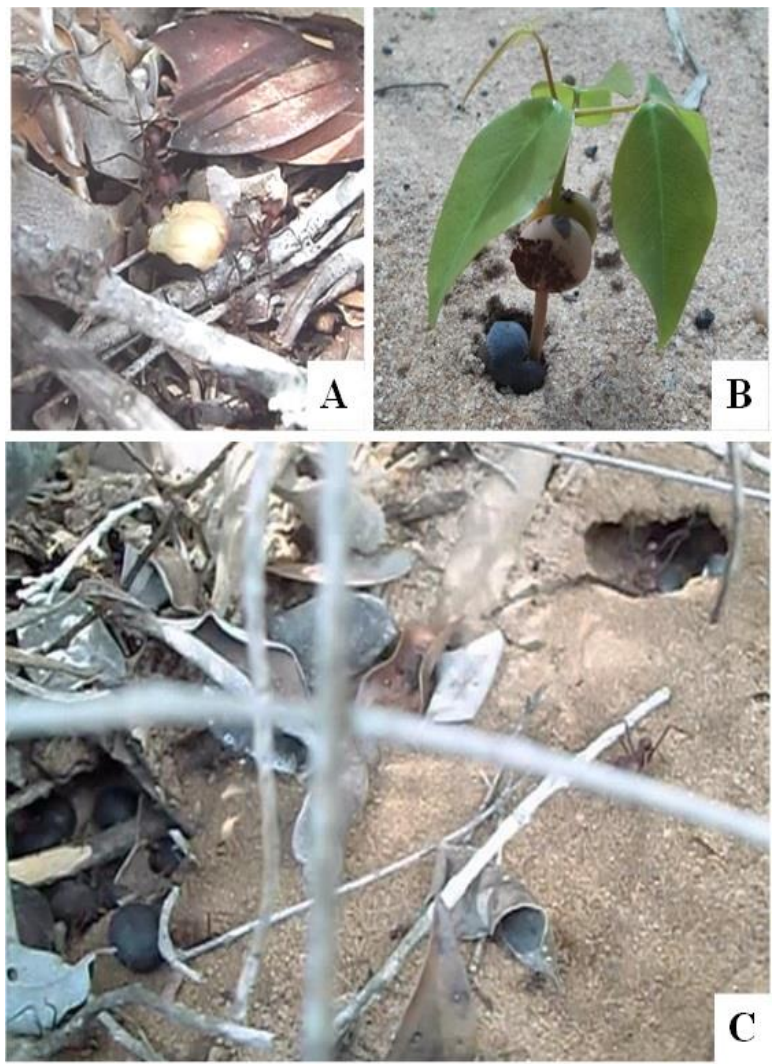

Figura 3. Copaifera arenicola (Ducke) J. Costa \& L. P Queiroz (Fabaceae) em remanescente de caatinga, município de Ribeira do Pombal-BA: A- Atta opacicens Boromeier. 1939 transportando sementes; B- Plântula de C. arenicola; C-Sementes alojadas em ninhos de $A$. opaciceps.
Em uma área no Cerrado, Lopes et al. (2018) estudando a dispersão de frutos e sementes de Simarouba versicolor A. St-Hil. (Simaroubaceae) por Atta sexdens (L.) ao longo de trilhas de forrageamento e no formigueiro de uma colônia, constataram a dispersão a 20 metros da área, onde as sementes limpas pelas formigas germinaram mais rapidamente. Também no Cerrado, Leal e Oliveira (1998) identificaram, dentre outras espécies de formigas dispersoras de sementes, as cortadeiras Atta spp. e Acromyrmex spp., transportando sementes a uma distância de cerca de $12 \mathrm{~m}$, as quais foram atraídas pelos elaissomos.

Adicionalmente, Farnese et al. (2011) constataram a presença de Atta spp. e Acromyrmex spp. interagindo com sementes sem elaissomos, com maior dispersão daquelas com maior proporção de arilo.

Ainda no Cerrado, Lima et al. (2013), encontraram formigas A. sexdens rubropilosa dispersando sementes e frutos de Miconia sp. (Melastomataceae) entre as distâncias de 0,1 a $45,2 \mathrm{~m}$. Os autores também notaram que a limpeza das sementes foi um importante serviço prestado pelas formigas favorecendo na germinação de sementes.

Silva e Souza (2014), no Cerrado, registraram formigas cortadeiras $A$. sexdens transportando sementes de $C$. langsdorffi Desf. até o formigueiro, atraídas pelo arilo presente nas sementes. Fato esse diferentemente notado no presente trabalho, onde, todas as sementes forrageadas por A. opaciceps até o formigueiro não continham arilo.

Silva e Souza (2014) ainda notaram que o tamanho robusto da $A$. sexdens facilitou o transporte das sementes para dentro dos ninhos, utilizando o arilo como suporte para o deslocamento das sementes, proporcionando uma melhor dispersão por percorrer longas distâncias já que encontraram sementes em olheiros com $4 \mathrm{~m}$ e até $80 \mathrm{~m}$ da planta-mãe. Fato esse também observado em estudo de Teixeira et al. (2007) em ambiente de Restinga, onde ocorreu interação de A. robusta com 35 sementes diferentes não mirmecóricas, com a dispersão observada em maior frequência e maior distância realizada apenas por formigas de grande porte. Teixeira et al. (2007) ainda observaram o ato de remoção dos arilos das sementes por formigas A. robusta Borgmeier, 1939 antes do transporte.

Em fragmentos vegetais no Bioma Mata Atlântica, Peternelli et al. (2004) identificaram formigas A. sexdens rubropilosa Forel, 1908 transportando sementes de Mabea fistulifera Mart. (Euphorbiaceae) por um percurso de $2 \mathrm{~m}$ a 4 $\mathrm{m}$, atraídos pelos elaissomos.

Essas observações prévias do comportamento das formigas descritas na literatura foram corroboradas pelos dados observados no presente trabalho, diferindo pelo fato das observações terem sido realizadas em ecossistema diferente, e das formigas não terem sido atraídas pelo arilo das sementes e nem elaissomos, uma vez que as sementes de C. arenicola não possuem elaissomos, e as sementes observadas no presente estudo já não possuíam arilo, o qual havia sido removido de alguma forma, e não pôde ser observada sua função na atratividade das formigas.

Nesse aspecto, as sementes vistas forrageadas por $A$. opaciceps, foram atraídas, provavelmente, pela amêndoa das sementes de $C$. arenicola, as quais tinham parte do tegumento retirado pelas formigas antes de serem transportadas ao ninho, fato esse ainda não encontrado em registro na literatura.

Viana-Bailez e Endringer (2016) explicam que formigas Atta spp. possuem um padrão de forrageamento bem definido por meio de trilhas físicas e químicas, podendo estar associado a fenômenos como o polimorfismo e o polietismo etário como característica dessas espécies, 
Gama et al.

variando o comportamento de forrageamento associado em função de fatores ambientais, espécies vegetais e da capacidade de adaptação.

\section{Conclusões}

A espécie de formiga Atta opaciceps demonstrou considerável potencial na dispersão de sementes de Copaifera arenicola, apesar do tamanho avantajado da semente. demonstrando seu importante papel nessas relações ecológicas em ambientes naturais, contrariando o seu efeito negativo quando atuam em desfolhamentos em culturas agrícolas, evidenciando a importância do manejo adequado desses insetos em áreas agrícolas, visando à conservação das interações ecológicas em áreas naturais do entorno dos cultivos.

\section{Agradecimentos}

Os autores são gratos ao Prof. Dr. Jacques H. C. Delabie (Laboratório de Mirmecologia, Departamento de Ciências Agrárias e Ambientais (DCAA), Universidade Estadual de Santa Cruz, Ilhéus, Bahia, Brasil) pela identificação das formigas.

\section{Referências}

Autuori M (2010). Investigações sobre a biologia da saúva. Ciência e Cultura, 62 (1): 4-12.

Corrêa MM, Silva PS, Wirth R, Tabarelli M, Leal IR (2010). How leaf-cutting ants impact forests: drastic nest effects on light environment and plant assemblages. Oecologia, 162(1): 103-115. doi.org/10.1007/s00442-009-1436-4.

Costa ÚASD, Oliveira M, Tabarelli M, Leal IR (2007). Dispersão de sementes por formigas em remanescentes de floresta atlântica nordestina. Revista Brasileira de Biociências, 5(1): 231-233.

Deminicis BB, Vieira HD, Araujo SAC, Jardim JG, Pádua FT, Chambela-Neto A (2009). Dispersão natural de sementes: importância, classificação e sua dinâmica nas pastagens tropicais. Archivos de Zootecnia, (58): 35-58.

Farnese FS, Campos RBF, Fonseca GA (2011). Dispersão de diásporos não mirmecóricos por formigas: influência do tipo e abundância do diásporo. Revista Árvore, 35(1): 125-130. doi.org/10.1590/S0100-67622011000100015.

Fernandes TV, Paolucci LN, Carmo FM, Sperber CF, Campos RI (2018). Seed manipulation by ants: disentangling the effects of ant behaviours on seed germination. Ecological entomology, 43(6):712-718.DOI: 10.1111/een.12655.

Gama DC, Nascimento Júnior JMD (2019). Copaifera arenicola [(Ducke) J. Costa e L. P. Queiroz] FabaceaeCaesalpinioideae em Regiões do Nordeste da Bahia. Agroforestalis News, 4(1): i-viii.

Gama DC, Jesus JBD (2018). Aspecto geomorfológico, hidroclimático e ambiental da microrregião de Ribeira do Pombal, Bahia, Brasil. Geoambiente On-Line, (32): 57-73. doi.org/10.5216/revgeoamb.v0i32.51034

Gorb SN, Gorb EV (1995). Removal rates of seeds of five myrmecochorous plants by the ant Formica polyctena (Hymenoptera: Formicidae). Oikos (73): 367-374. Doi.10.2307/3545960.

Hardesty BD (2011). Effectiveness of seed dispersal by ants in a neotropical tree. Integrative zoology, 6(3): 222-226. doi: $10.1111 / \mathrm{j} .1749-4877.2011 .00246 . x$
Hölldobler B, Wilson EO (1990). The ants. Harvard: University Press. 732p.

Leal IR, Oliveira PS (1998). Interactions between fungusgrowing ants (Attini), fruits and seeds in cerrado vegetation in southeast Brazil. Biotropica. (30): 170-178. doi:10.1111/j.1744-7429.1998.tb00052.x

Leal IR, Lopes AV, Machado IC, Tabarelli M (2018). Interações planta-animal na Caatinga: visão geral e perspectivas futuras. Ciência e Cultura, 70(4): 35-40.

Leal IR, Wirth R, Tabarelli M (2007). Seed dispersal by ants in the semi-arid Caatinga of Northeast Brazil. Annals of Botany, 99(5): 885-894. DOI.org/10.1093/aob/mcm017.

Lima MH, Oliveira EG. Silveira FA (2013). Interactions between ants and non-myrmecochorous fruits in Miconia (Melastomataceae) in a neotropical savanna. Biotropica, 45(2):217-223.doi.org/10.1111/j.1744-7429.2012.00910.x.

Lopes DRS, Tavares RC, Batista KOM, Souza PBD, Nascimento MOD, Souza DJD (2018). Simarouba versicolor (Simaroubaceae) Dispersal by the Leaf-Cutter Ant Atta sexdens. Sociobiology, 65(2): 337-339. DOI:10.13102/sociobiology.v65i2.2162.

Meyer ST, Leal IR, Tabarelli M, Wirth R (2011). Ecosystem engineering by leaf-cutting ants: nests of Atta cephalotes drastically alter forest structure and microclimate. Ecological Entomology, 36(1): 14-24.DOI: 10.1111/j.1365-2311.2010.01241.x.

Moutinho P, Nepstad DC, Davidson EA (2003). Influence of leaf-cutting ant nests on secondary forest growth and soil properties in Amazonia. Ecology, (84):1265-1276. doi10.1890/00129658(2003)084[1265:IOLANO]2.0.CO;2

Oliveira MAD, Gomes CFF, Pires EM, Marinho CGS, Della Lucia TMC (2014) Bioindicadores ambientais: insetos como um instrumento desta avaliação. Ceres, 61(7): 800807. DOI.org/10.1590/0034-737X201461000005.

Pedroni F, Sanchez M, Santos FAM (2002). Fenologia da copaíba (Copaifera langsdorffii Desf. - Leguminosae, Caesalpinioideae) em uma floresta semidecídua no sudeste do Brasil. Revista Brasileira de Botânica, 25(2): 183-194. doi.org/10.1590/S0100-84042002000200007.

Peternelli EFO, Della-Lucia TMC, Martins SV (2004). Espécies de formigas que interagem com as sementes de Mabea fistulifera Mart. (Euphorbiaceae). Revista Árvore, 28(5): 733-738

Queiroz JM, Almeida FS, Pereira MPS (2006). Conservação da biodiversidade e o papel das formigas. Floresta e ambiente, 13(2): 37-45.

Teixeira MC (2007). Dispersão de sementes por Atta robusta Borgmeier 1939 (Hymenoptera: Formicidae) nas restingas da Ilha de Guriri-ES. Viçosa: Universidade Federal de Viçosa. 72 p. Tese de Doutorado.

Viana-Bailez AM, Endringer FB (2016). "Plasticidade do comportamento de forrageamento em formigas cortadeiras." Oecologia Australis, 20(3): 11-19. doi.org/10.4257/oeco.2016.2003.02.

Silva A, Souza DJD (2014). Interação entre Atta sexdens e espécie arbórea Copaifera langsdorfii Desf. em remanescente florestal de cerrado. Revista Verde, 9(3): 182-189. 